Supporting Information for

\title{
Continuous, Ultra-lightweight, and Multipurpose
}

\section{Super-aligned Carbon Nanotube Tapes Viable over a}

\section{Wide Range of Temperatures}

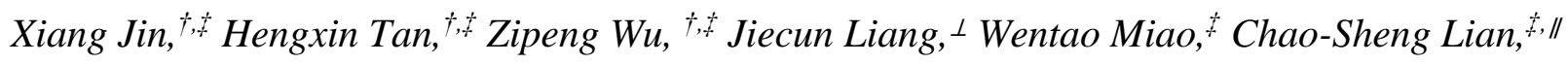

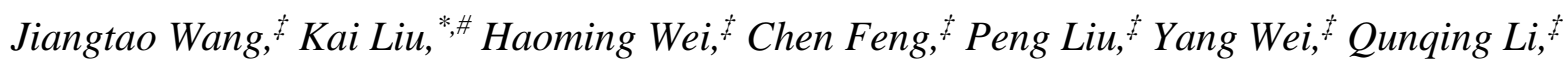

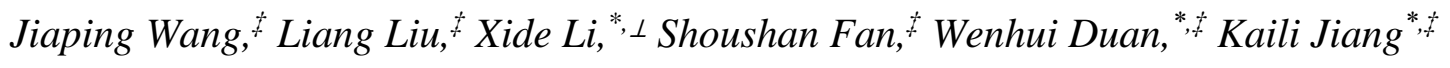

State Key Laboratory of Low-Dimensional Quantum Physics, Department of Physics and

Tsinghua-Foxconn Nanotechnology Research Center, Tsinghua University, Beijing 100084,

China.

${ }^{\perp}$ School of Aerospace Engineering, Tsinghua University, Beijing 100084, China.

"International Laboratory for Quantum Functional Materials of Henan and School of Physics and Engineering, Zhengzhou University, Zhengzhou 450001, China.

\#State Key Laboratory of New Ceramics and Fine Processing, School of Materials Science and Engineering, Tsinghua University, Beijing 100084, China. 


\section{Derivation of Eq. 1}

In Eq. $1, F_{0}$ represents the areal adhesive strength of a SACNT tape. After one cycle of detachment and re-attachment, $p_{1}\left(0<p_{1}<1\right)$ is the Areal fraction of the SACNT tape that is disrupted and the adhesive force is reduced to $t F_{0}(0<\mathrm{t}<1)$. Eq. 1 can be derived from the following process:

$$
\begin{aligned}
& 1^{\text {st }} \text { cycle: } \quad F_{1}=F_{0} \\
& 2^{\text {nd }} \text { cycle: } F_{2}=F_{0} \cdot\left(1-p_{1}\right)+t F_{0} \cdot p_{1} \\
& 3^{\text {rd }} \text { cycle: } F_{3}=F_{0} \cdot\left(1-p_{1}\right)^{2}+t F_{0} \cdot\left(1-\left(1-p_{1}\right)^{2}\right) \\
& =F_{0} \cdot\left(t+(1-t)\left(1-p_{1}\right)^{2}\right) \\
& \mathrm{n}^{\text {th }} \text { cycle: } \quad F_{n}=F_{0} \cdot\left(1-p_{1}\right)^{n-1}+t F_{0} \cdot\left(1-\left(1-p_{1}\right)^{n-1}\right) \\
& =F_{0} \cdot\left(t+(1-t)\left(1-p_{1}\right)^{n-1}\right)
\end{aligned}
$$

Table S1. Fitted parameters of Eq. 1 for the cyclic tests. Cyclic tests were done for different areas using the same sample, an 8-layer $\mathrm{SACNT}$ tape on a $\mathrm{SiO}_{2} / \mathrm{Si}$ substrate, yielding data of the adhesive strength $v s$. the number of cycles.

\begin{tabular}{|c|c|c|}
\hline$F_{0}(\mathrm{~N})$ & $t$ & $p_{1}$ \\
\hline 0.73924 & 0.49716 & 0.49527 \\
\hline 0.78679 & 0.50061 & 0.44078 \\
\hline 0.61869 & 0.51333 & 0.51028 \\
\hline 0.64645 & 0.4407 & 0.49321 \\
\hline 0.59348 & 0.46513 & 0.50331 \\
\hline 0.73794 & 0.52134 & 0.47902 \\
\hline 0.69393 & 0.44835 & 0.43741 \\
\hline
\end{tabular}




\section{Derivation of Eq. 2}

In Eq. $2, F_{\mathrm{s}}$ represents the saturated areal adhesive force, $l$, the number of SACNT tape layers, and $p_{2}$, the fractional increase in the effective area contributing to the vdW forces if an additional SACNT tape layer is added onto the surface. Eq. 2 can be derived from the following process:

$$
\begin{aligned}
& 1^{\text {st }} \text { layer: } F_{1}=F_{S} \cdot p_{2} \\
& 2^{\text {nd }} \text { layer: } F_{2}=F_{S} \cdot p_{2}+F_{s} \cdot\left(1-p_{2}\right) \cdot p_{2} \\
& 3^{\text {rd }} \text { layer: } F_{3}=F_{S} \cdot p_{2}+F_{S} \cdot\left(1-p_{2}\right) \cdot p_{2}+F_{S} \cdot\left(1-p_{2}\right)^{2} \cdot p_{2} \\
& \qquad=F_{S} \cdot\left(1-\left(1-p_{2}\right)^{3}\right) \\
& \ldots \ldots \\
& l^{\text {th }} \text { layer: } F_{l}=F_{S} \cdot\left(1-\left(1-p_{2}\right)^{l}\right)
\end{aligned}
$$

Table S2. Fitted parameters of Eq. 2 on different surfaces. Data of adhesive strength $v s$. the number of SACNT layers were used for the fitting.

\begin{tabular}{|c|c|c|c|c|}
\hline & \multicolumn{2}{|c|}{$F_{\mathrm{S}}\left(\mathrm{N} / \mathrm{cm}^{2}\right)$} & \multicolumn{2}{c|}{$p_{2}$} \\
\hline & Value & Standard error & Value & Standard error \\
\hline Rough $\mathrm{Si}_{3} \mathrm{~N}_{4}$ & 1.98 & 0.06 & 0.081 & 0.005 \\
\hline Smooth $\mathrm{Si}_{3} \mathrm{~N}_{4}$ & 4.7 & 0.1 & 0.17 & 0.02 \\
\hline $\mathrm{Si}$ & 4.4 & 0.1 & 0.122 & 0.009 \\
\hline Acrylic glass & 3.1 & 0.1 & 0.13 & 0.01 \\
\hline $\mathrm{Cu}$ & 1.3 & 0.1 & 0.42 & 0.07 \\
\hline $\mathrm{Al}$ & 1.80 & 0.04 & 0.63 & 0.01 \\
\hline
\end{tabular}




\section{Raman spectrum of SACNT tapes}

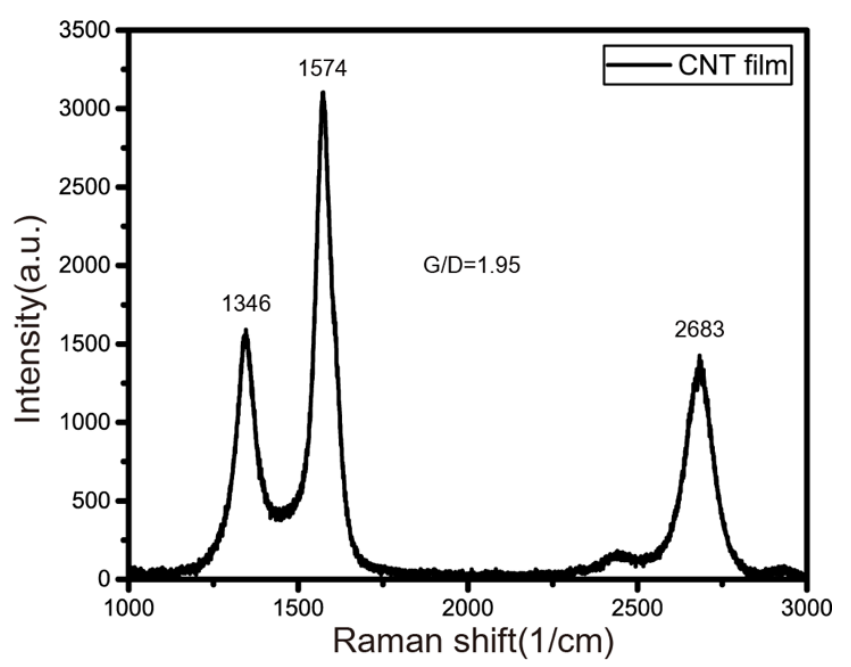

Figure S1. Raman spectrum of a SACNT tape, measured at an excitation wavelength of $514 \mathrm{~nm}$. It shows a G/D ratio of 1.95 .

\section{Force $v s$. displacement curves for single-sided \& double-sided SACNT tapes}
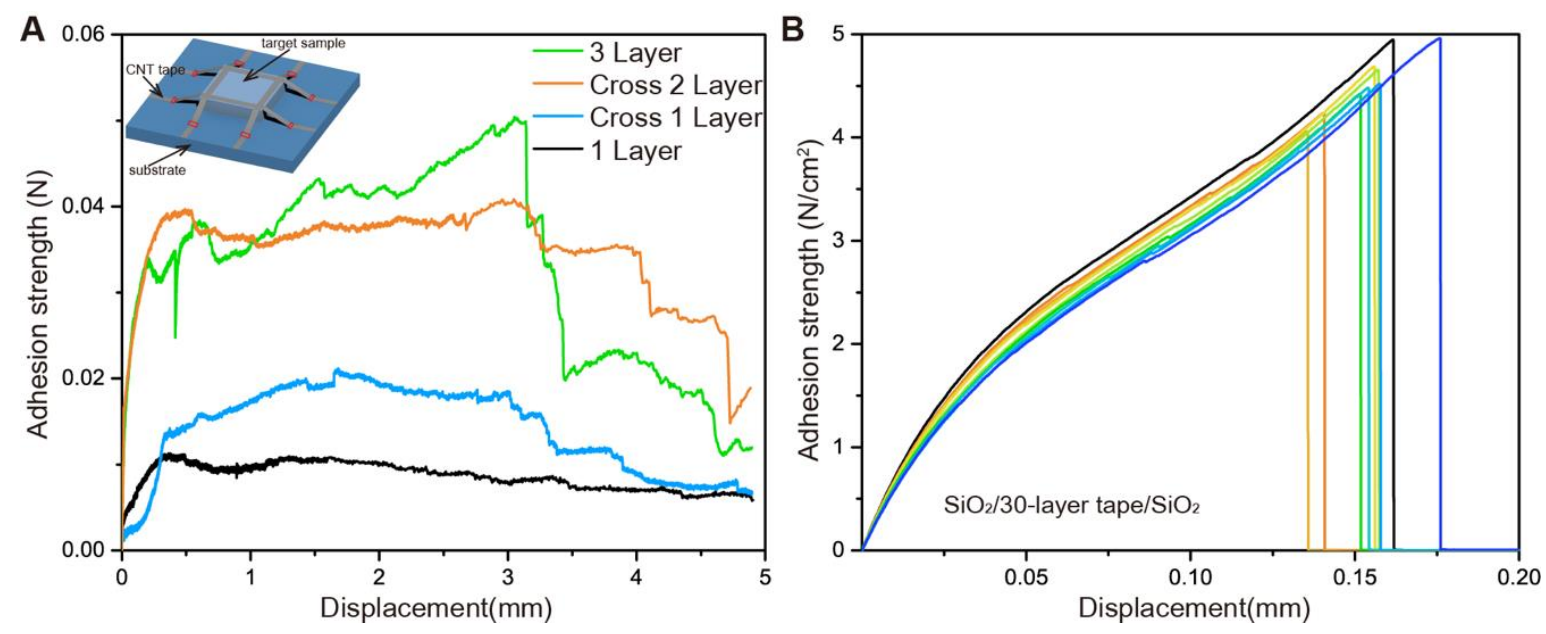

Figure S2. Curves of normal adhesive strength $v s$. displacement in single-sided mode (A) and in double-sided mode (B). The inset in (A) shows the method used to adhere a SACNT tape in single-sided mode. In (A), for the black curve labeled as '1 Layer', two strips of SACNT tape each about $3 \mathrm{~mm}$ wide were used to bind a sample $(2 \mathrm{~cm} \times 2 \mathrm{~cm})$ to the substrate $(5 \mathrm{~cm} \times 5 \mathrm{~cm})$; for the blue curve labeled as 'Cross 1 Layer', two additional strips of SACNT tape positioned 
perpendicular to the first two were also used. Labeling for the other curves is self-explanatory. Ethanol was applied to reduce the distance between the CNTs and the substrate.

Figure S2 shows that the adhesive strength in single-sided mode is much smaller than that in double-sided mode, but SACNT tape in single-sided mode has a far wider working displacement range. The reason behind this phenomenon is that, in single-sided mode the SACNT tape is being peeled off from the substrate gradually during the detachment process, while in doublesided mode detachment occurs collectively all at once. Thus, in single-sided mode, only small portions of the SACNT tapes - red rectangular areas in insert of Figure S2A — contribute to adhesion. This is much less than the effective adhesive area in double-sided mode.

\section{SACNT tapes for wafer-scale adhesion}
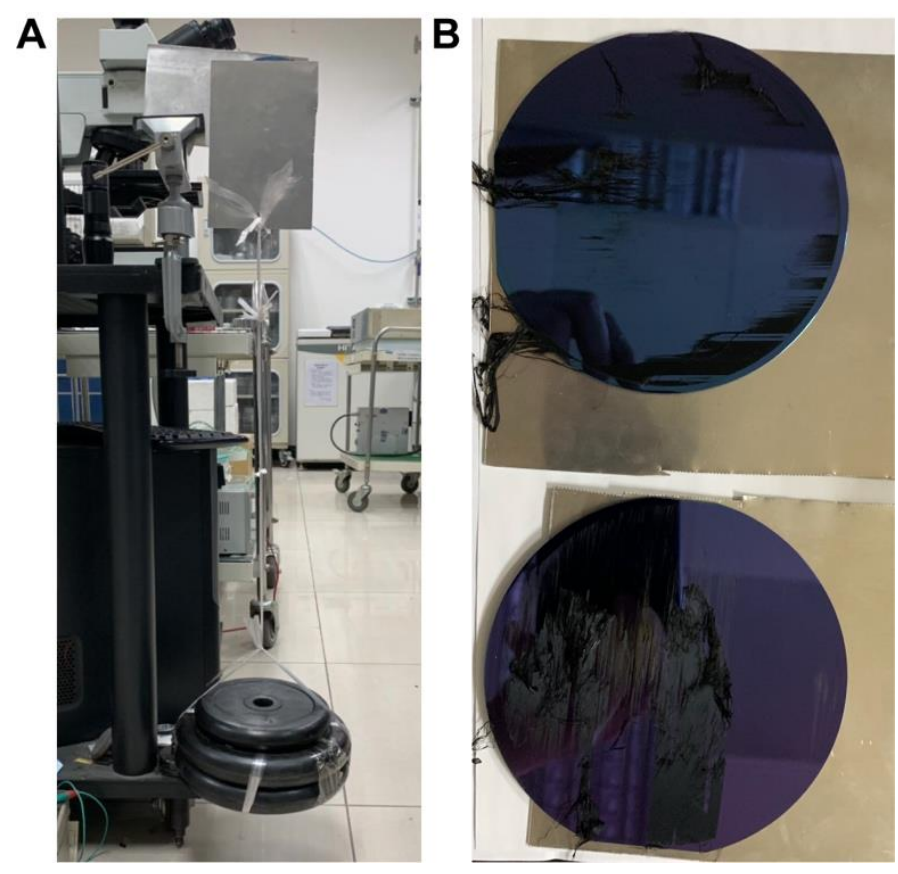

Figure S3. SACNT tapes for wafer-scale adhesion. (A) Side-view photograph showing a $10.5 \mathrm{~kg}$ weight loaded on a 4 inch silicon wafer via an SACNT tape. (B) Top-view photograph showing the morphology of wafers after destruction in two separate experiments. 


\section{Setup for high-temperature measurements}
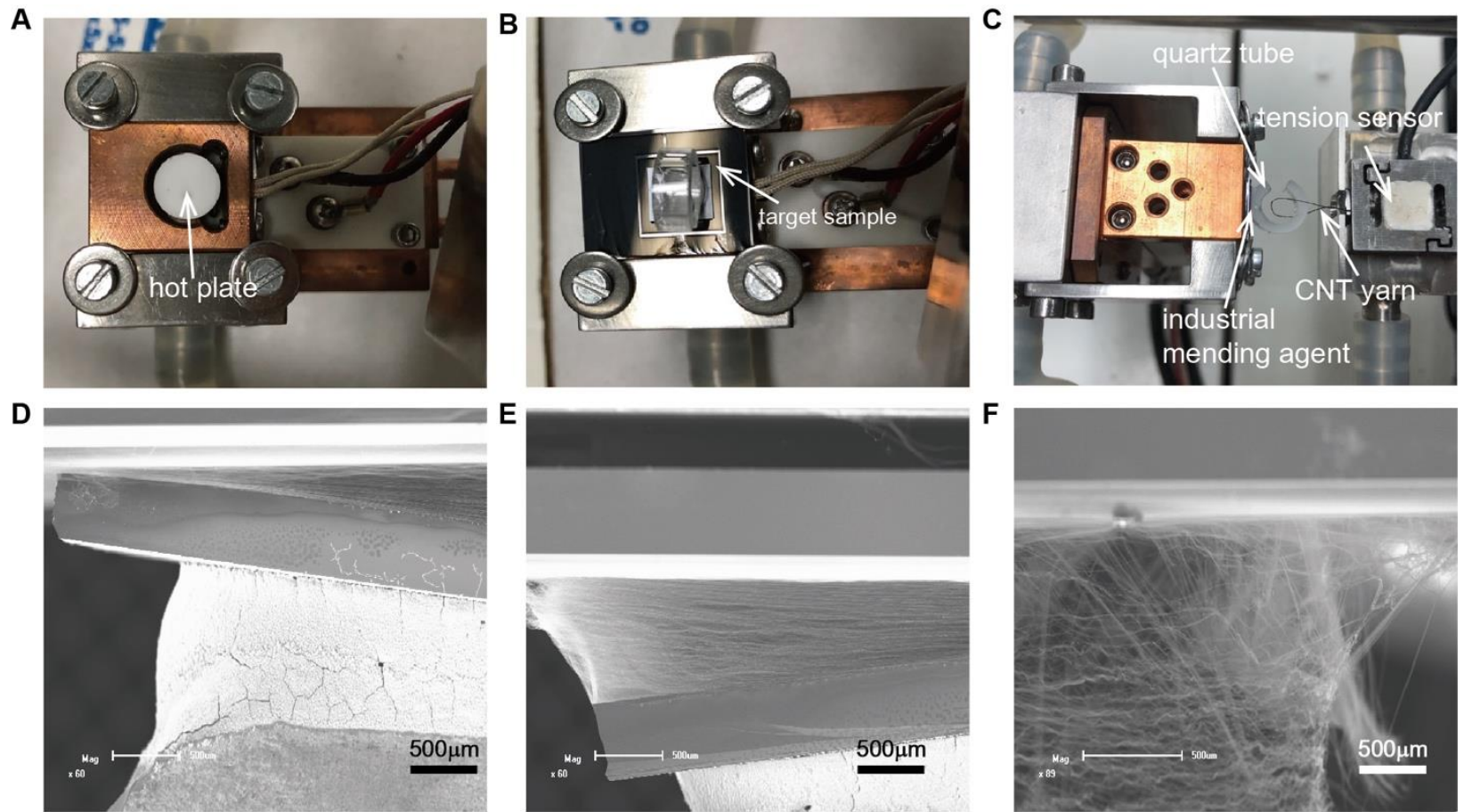

$\mathbf{F}$

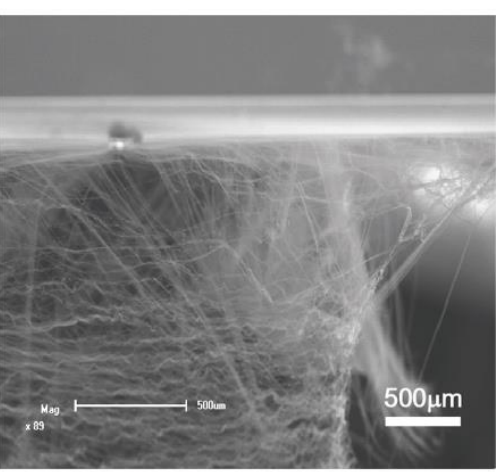

G
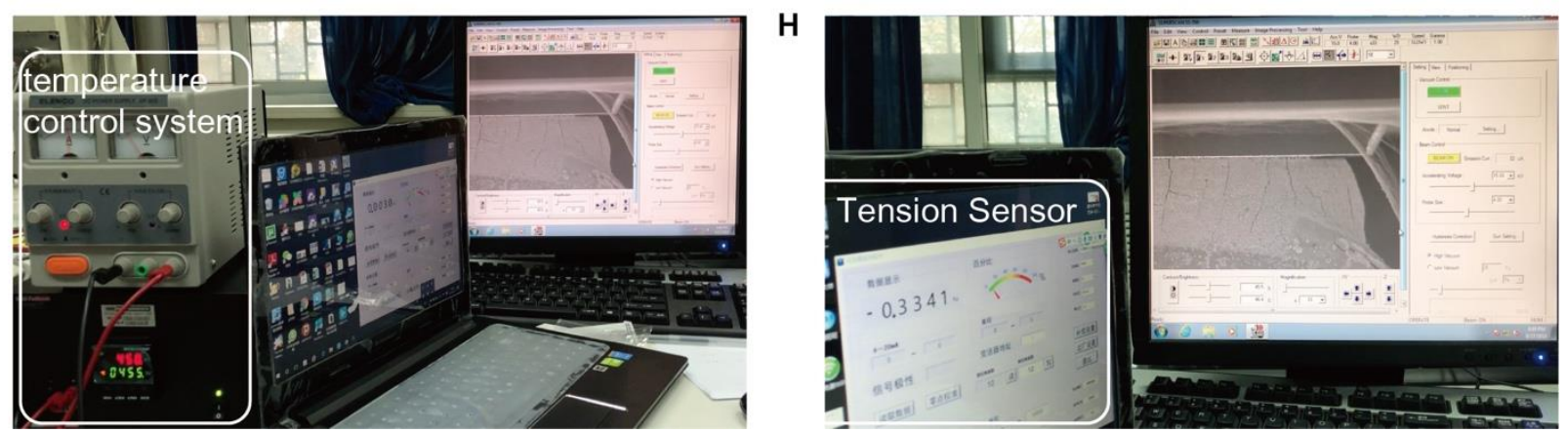

Figure S4. Setup for high-temperature measurements. (A) Top-view photograph of the small hot plate which can be heated to $1000{ }^{\circ} \mathrm{C}$ in a vacuum. The hot plate can be placed inside an SEM chamber for in-situ measurements. (B) Top-view photograph of a sample fixed onto the hot plate. The sample contains two substrates adhered together by a SACNT tape. (C) Side-view photograph of the measurement setup, the sample is bonded to a quartz tube bent into a partial ring (with a $\sim 45^{\circ}$ gap) on one side and connected to a tension sensor via CNT yarn on the other. With this setup, force $v s$. displacement curves of samples were recorded at various temperatures in a vacuum in the SEM chamber. (D-F) SEM pictures showing the detachment process caused by 
an increasing tensile load. (G-H) Photographs showing the apparatus used to control temperatures and to measure adhesive forces.

\section{Comparison of adhesive strengths for parallel-stacking, cross-stacking, and alcohol- shrinking SACNT tapes}

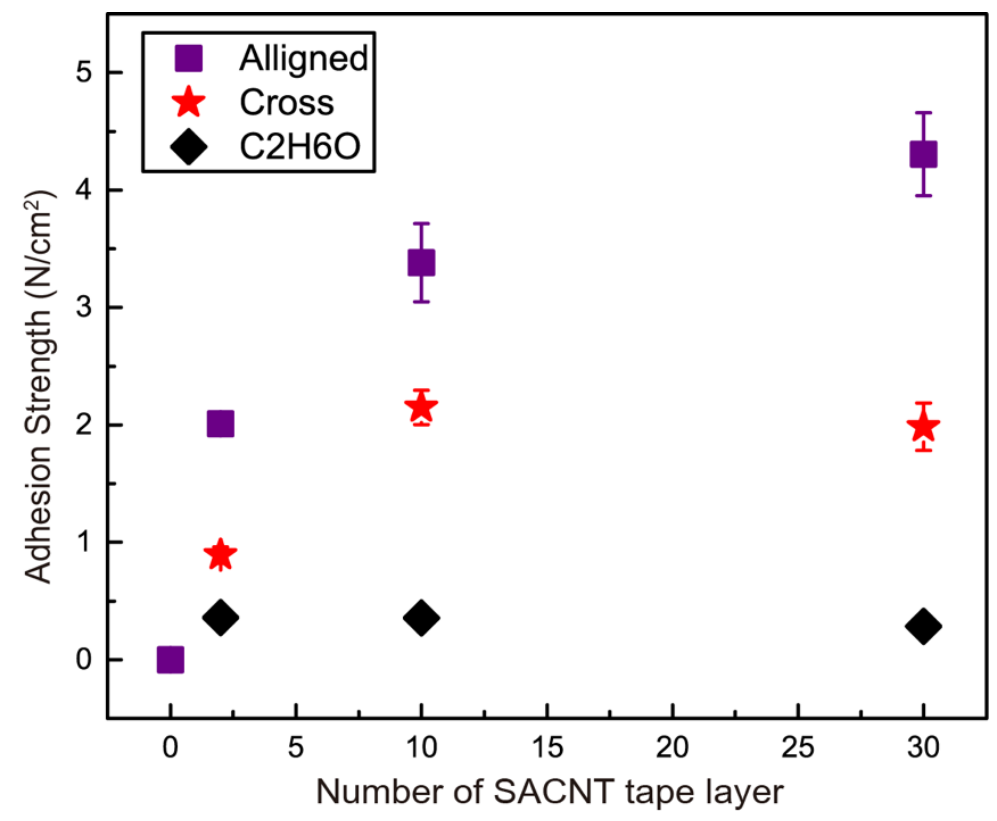

Figure S5. Comparison of adhesive strengths of SACNT tapes with three different treatments: parallel-stacking, cross-stacking, and alcohol-shrinking. Parallel-stacked SACNT tape clearly has the biggest adhesive strength. This is because it has more surface CNTs that can contact the surface of the substrate. If SACNT tapes are cross-stacked layer by layer, the CNTs in the upperlayer SACNT tape cannot fill the gaps left by the layer below, reducing the number of CNTs within range of the vdW force. The adhesive strength is saturated when ten layers of SACNT tapes are cross-stacked over the surface. If parallel-stacked SACNT tapes are applied with droplets of alcohol, the tapes will shrink into large bundles after the alcohol evaporates. This greatly increases the surface roughness of the SACNT tapes, resulting in a weak adhesive strength. 


\section{Setup for measurements at liquid-nitrogen temperature}

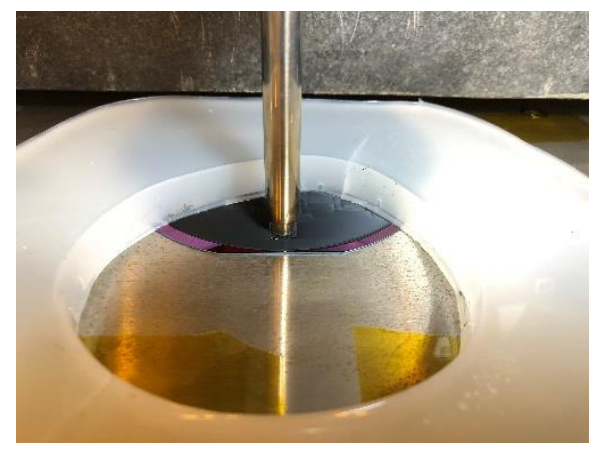

Figure S6. Setup for measurements at liquid-nitrogen temperature. The sample is immersed into liquid nitrogen in a pool surrounded by a silica gel.

\section{Deterioration mode of SACNT tape}

As observed in SEM images (Fig. 3C), the failure of the adhesion should be attributed mainly to the detachment of CNTs from the SACNT tape and partly to the breakdown of the nodes that connect adjacent CNT bundles. We illustrated the mechanism in Fig. S7.
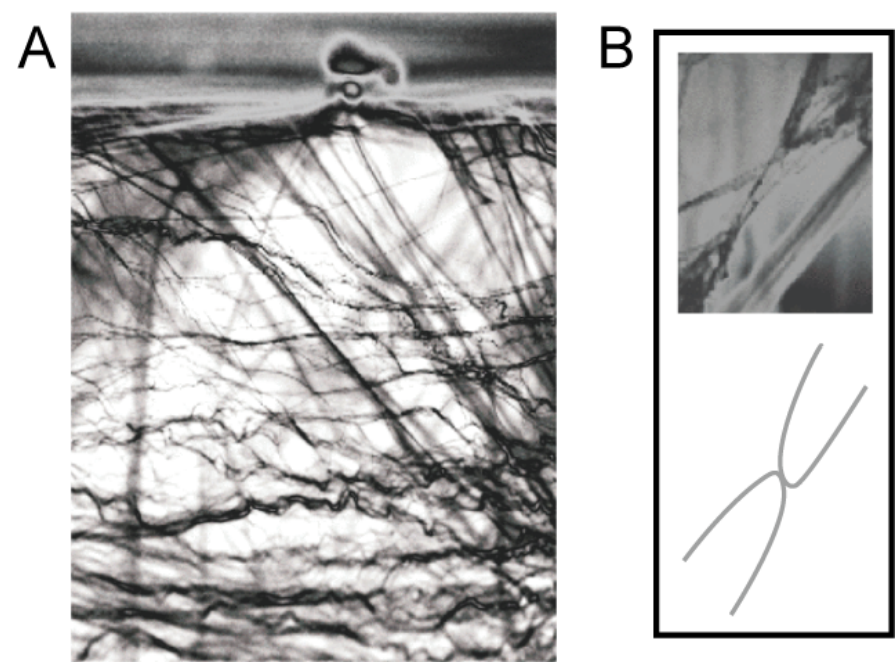

Figure S7. (A) False-colored image of SACNT tape after deterioration. (B) Locally enlarged image and schematic showing the breakdown of the nodes that connect adjacent CNT bundles. 


\section{Relationship between mechanical strength (modulus) and adhesion strength}

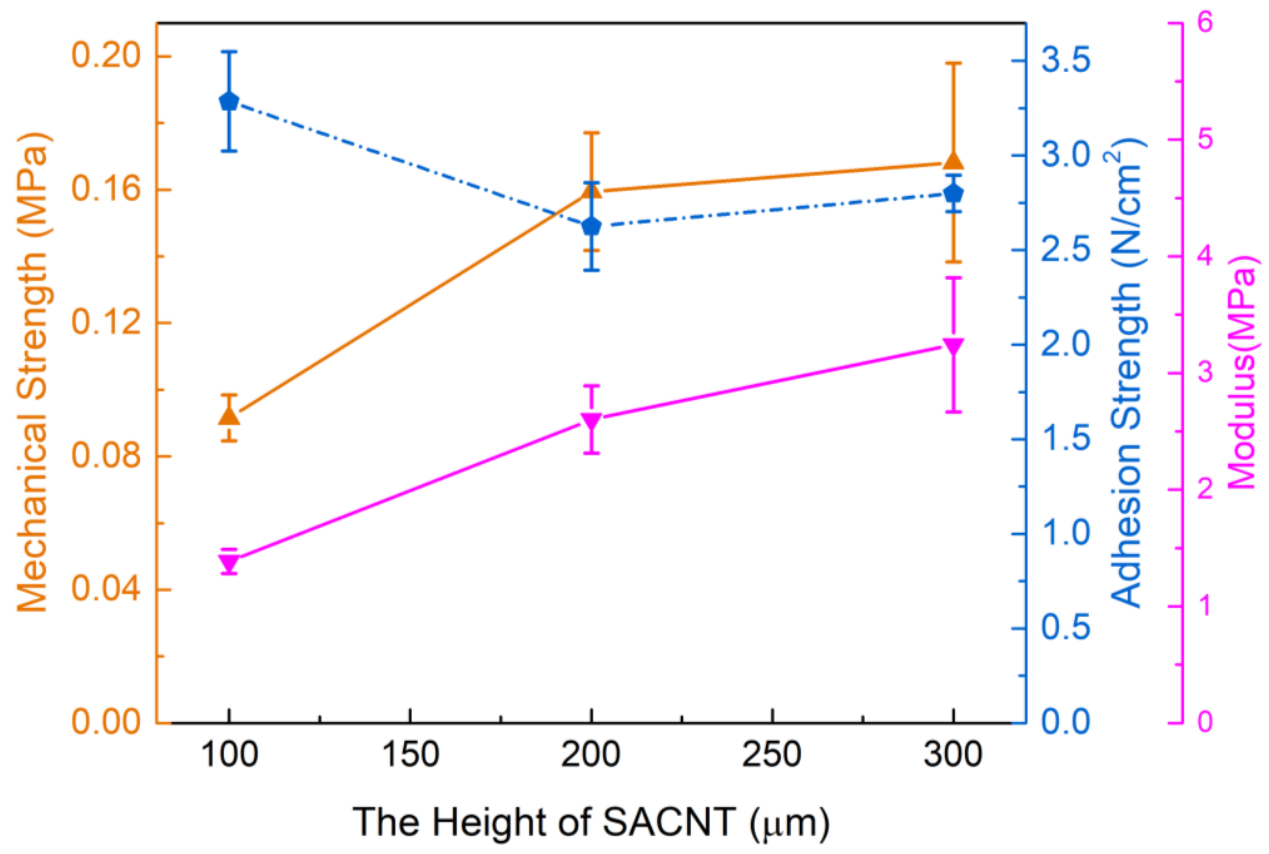

Figure S8. Adhesion strength and mechanical strength of SACNT tapes which are from three different height of SACNT. The data is based on 10-layer SACNT film.

As shown in Fig. S8, both the tensile strength and the modulus is the lowest for the SACNT tape drawn from the shortest SACNT array. However, the adhesion strength is the largest for this film. It suggests that the adhesion strength of the SACNT tapes does not much depend on the mechanical properties of the SACNT film. We think that the adhesion strength of the tapes should be mainly dominated by their surface roughness instead of their mechanical strengths. The as-drawn SACNT film drawn from a thick SACNT array usually consists of large CNT bundles and thus has a large surface roughness (Nano Lett. 2008, 8, 700), leading to a low adhesion strength. 
Table S3. Simulation parameters based on numerical calculations. Results are discussed in some detail under the heading Simulations in the paper.

\begin{tabular}{|c|c|c|c|c|c|c|}
\hline Surface & CNT & $\begin{array}{c}\text { Diameter } \\
(\AA)\end{array}$ & $\begin{array}{l}\text { Mismatch of } \\
l \text { and } a(\%)\end{array}$ & $\begin{array}{l}\text { Equilibrium } \\
\text { Distance (̊) }\end{array}$ & $\begin{array}{l}\text { Distance } \\
\text { of Max. } \\
\text { Force }(\AA)\end{array}$ & $\begin{array}{l}\text { Max. Force } \\
\left(1^{4} \mathbf{N} / \mathbf{c m}^{2}\right)\end{array}$ \\
\hline \multirow{5}{*}{$\mathrm{SiO}_{2}(001)$} & $(6,6)$ & 8.14 & 0.1 & 2.67 & 3.26 & 7.6 \\
\hline & $(8,8)$ & 10.85 & 0.1 & 2.69 & 3.27 & 6.7 \\
\hline & $(10,10)$ & 13.56 & 0.1 & 2.69 & 3.27 & 5.5 \\
\hline & $(12,12)$ & 16.27 & 0.1 & 2.62 & 3.20 & 5.1 \\
\hline & $(17,0)$ & 13.31 & 0.1 & 2.70 & 3.27 & 5.3 \\
\hline \multirow{5}{*}{$\mathrm{Cu}(111)$} & $(6,6)$ & 8.14 & -3.8 & 2.88 & 3.65 & 23.6 \\
\hline & $(8,8)$ & 10.85 & -3.8 & 2.86 & 3.62 & 19.8 \\
\hline & $(10,10)$ & 13.56 & -3.8 & 2.88 & 3.61 & 17.0 \\
\hline & $(12,12)$ & 16.27 & -3.8 & 2.86 & 3.60 & 15.3 \\
\hline & $(17,0)$ & 13.31 & -3.8 & 2.84 & 3.61 & 18.0 \\
\hline \multirow{4}{*}{ Ag (111) } & $(6,6)$ & 8.14 & -1.7 & 3.03 & 3.83 & 20.1 \\
\hline & $(8,8)$ & 10.85 & -1.7 & 2.98 & 3.73 & 16.7 \\
\hline & $(10,10)$ & 13.56 & -1.7 & 3.03 & 3.75 & 15.2 \\
\hline & $(12,12)$ & 16.27 & -1.7 & 3.00 & 3.72 & 13.3 \\
\hline \multirow{4}{*}{$\mathrm{Au}(111)$} & $(6,6)$ & 8.14 & -1.5 & 3.11 & 3.81 & 22.1 \\
\hline & $(8,8)$ & 10.85 & -1.5 & 3.09 & 3.81 & 18.4 \\
\hline & $(10,10)$ & 13.56 & -1.5 & 3.12 & 3.74 & 16.0 \\
\hline & $(12,12)$ & 16.27 & -1.5 & 3.10 & 3.73 & 14.5 \\
\hline
\end{tabular}

\section{Movie Captions}

Movie S1: The SACNT tape can be drawn in liquid nitrogen $\left(-196^{\circ} \mathrm{C}\right)$.

Movie S2: The SACNT tape can be drawn at a high temperature $\left(1200^{\circ} \mathrm{C}\right)$. 